\title{
FIABILIDAD Y EVIDENCIAS DE VALIDEZ DE UN INSTRUMENTO PARA LA EVALUACIÓN DE LA CALIDAD DE LOS MAPAS CONCEPTUALES
}

\author{
Beatriz Lucas-Molina \\ Universidad de Valencia \\ Alicia Pérez-Albéniz Iturriaga \\ Eduardo Fonseca-Pedrero \\ Javier Ortuño-Sierra \\ María Luz Urraca \\ Marta Santarén-Rosell \\ Universidad de La Rioja
}

\begin{abstract}
RESUMEN: Diversos autores han señalado el interés y la pertinencia de los mapas conceptuales como herramientas para el aprendizaje significativo. Sin embargo, la revisión de la literatura pone de manifiesto la escasez de propuestas dirigidas a la evaluación de los mapas con criterios objetivables y la complejidad de las existentes. El objetivo del presente estudio fue desarrollar un instrumento para la evaluación de los mapas conceptuales en formato rúbrica. De esta manera, se consigue que los alumnos posean una herramienta que permita, mediante indicadores precisos, la evaluación de la calidad tanto de sus ejecuciones como de las ejecuciones realizadas por otros. Se construyó una rúbrica que incluye los criterios más aceptados en la literatura para la evaluación de mapas conceptuales. Se realizaron dos estudios piloto con sendos grupos de alumnos universitarios ( $n=31$ y $n=18$ ) que recibieron un programa de entrenamiento de cuatro horas. Los resultados indicaron que el instrumento diseñado muestra una buena fiabilidad interjueces e indicadores de validez discriminante. Se concluye que el instrumento diseñado para la evaluación de la calidad de los mapas conceptuales en formato rúbrica muestra adecuadas propiedades psicométricas. Asimismo, el instrumento permite aplicar criterios objetivables en la evaluación de los mapas y la comparación entre diferentes ejecuciones.
\end{abstract}

PALABRAS CLAVE: Mapas conceptuales, evaluación, intervención, instrumento. 


\title{
RELIABILITY AND SOURCES OF VALIDITY EVIDENCES FOR AN INSTRUMENT ASSESSING THE QUALITY OF CONCEPT MAPS
}

\begin{abstract}
Several authors have noticed the interest and relevance of concept mapping as a tool for promoting meaningful learning. However, the current literature highlights the lack of procedures for the assessment of concept mapping using objective criteria, as well as the complexity of the existing ones. The aim of this study was to develop an instrument for assessing concept maps through a rubric format. It is expected to provide the students with a tool that allows, through precise indicators, the assessment of the quality of their and others' performance. A rubric that includes the most widely accepted criteria in the literature is presented and examined. Two pilot studies with a group of pre-service teacher students each one ( $n=31$ and $n=18$, respectively) were conducted through a four hours training program. The results indicated that the designed instrument shows good indicators of interrater reliability and discriminant validity. It is then concluded that the designed instrument shows adequate psychometric properties. The instrument allows both the use of objective criteria in the assessment of concept maps, and the comparison between different executions.
\end{abstract}

KEYWORDS: Concept map, assessment, intervention, instrument.

Recibido: 05/07/2016

Aceptado: 07/09/2016

Correspondencia: Alicia Pérez de Albéniz Iturriaga, Universidad de La Rioja, Departamento de Educación. C/Luis de Ulloa, Edificio Vives, 26004 Logroño. Email: alicia. perez@unirioja.es.

\section{INTRODUCCIÓN}

Los mapas conceptuales surgieron en el marco de la teoría del aprendizaje significativo (Ausubel, Novak, Hanesian, 1987) como elementos articuladores de sus principios teóricos y, en consecuencia, como agentes eficaces del aprendizaje significativo. Ideados por Novak y sus colaboradores (Novak y Gowin, 1988) a mediados de los años setenta, los mapas conceptuales son instrumentos para organizar y representar el conocimiento.

Incluyen conceptos y términos de enlace. Los conceptos representan las regularidades que percibimos en los acontecimientos y en los objetos que nos rodean. Los términos de enlace conectan dos conceptos para formar proposiciones, que son la expresión de los significados que los alumnos atribuyen a la relación entre conceptos.

Por tanto, los mapas conceptuales tienen por objeto representar relaciones significativas entre conceptos en forma de proposiciones (Novak, 1998; Novak y Gowin, 1988). La mayor parte de los significados conceptuales se aprende mediante la composición de proposiciones en las que se incluye el concepto que se va a adquirir. Las proposiciones dan lugar a un incremento y precisión en el significado. Los mapas 
conceptuales dirigen la atención, tanto del estudiante como del profesor, sobre el reducido número de ideas importantes en las que deben concentrarse en cualquier tarea específica de aprendizaje y ofrecen un método visual para ayudar a los alumnos a organizar su propio pensamiento (Reyes, 2005, p. 16).

Además, es indudable que en el proceso de elaboración de los mapas se pueden desarrollar nuevas relaciones conceptuales, en especial si, de una manera activa, se trata de construir relaciones proposicionales entre conceptos que previamente no se consideraban relacionados. Novak y Gowin (1988) destacan que los estudiantes y profesores que elaboran mapas conceptuales señalan a menudo que se dan cuenta de nuevas relaciones y, por consiguiente, nuevos significados (o al menos significados que no poseían de una manera consciente antes de elaborar el mapa). En este sentido, la elaboración de mapas conceptuales puede ser una actividad creativa y puede ayudar a fomentar la creatividad.

Unido a lo anterior, se ha planteado que la utilización de mapas conceptuales no solo tiene evidentes implicaciones en el plano cognitivo por su potencial para la mejora de los procesos de aprendizaje sino que sus ventajas se extienden al plano emocional. Autores como Luque, Molina y Ontoria (2003) pusieron de manifiesto su impacto en aspectos tan relevantes como la autoestima o la satisfacción por aprender.

Una última ventaja a resaltar de los mapas conceptuales es que permiten detectar los denominados errores conceptuales, concepciones alternativas, jerarquías proposicionales inadecuadas, etc., que forman parte de la estructura cognitiva del alumno. A través de los errores conceptuales, el alumno interpreta la nueva información y esto le conduce a interpretaciones erróneas (González, 1997; González e Iraizoz, 1995; González, Morón y Novak, 2001) que desvirtúan la realidad. Los mapas conceptuales permiten hacer explícitos estos errores y, tras su reconocimiento, es posible promover el cambio conceptual.

El uso de los mapas conceptuales como herramienta para fomentar el aprendizaje significativo no es una estrategia novedosa. Novak et al. (1980) diseñaron el programa Aprendiendo a Aprender, basado en la teoría de Ausubel (1978), para su utilización simultánea junto con los programas regulares de instrucción mostrando resultados muy positivos. Asimismo, su utilización en la educación se ha generalizado en algunos países. Por ejemplo, en el 2005 se puso en marcha en Panamá el Proyecto Conéctate al Conocimiento (Tarté, 2006), un proyecto de transformación fomentado por el Ministerio de Educación en este país que contempla, entre otras cosas, la formación de docentes de centros de Educación Primaria en el uso de mapas conceptuales con sus alumnos.

Es por tanto evidente el interés y la pertinencia de los mapas conceptuales no sólo como herramientas para el aprendizaje significativo en estudiantes universitarios sino también su potencial de aplicación en otros ámbitos de aprendizaje (especialmente, con niños de Educación Infantil y Primaria, tal y como proponen autores como Ontoria et al., 1992). Dado que el equipo docente del presente estudio estaba interesado en introducir el mapa conceptual como estrategia de aprendizaje con los alumnos, se desarrolló y evaluó un programa (Pérez-Albéniz, Lucas-Molina, Solbes-Canales, Calderón y Martín-Seoane, 2016) para la enseñanza de la elaboración y evaluación de mapas conceptuales. 
Sin embargo, la evaluación de la calidad de los mapas conceptuales no es una tarea fácil. La revisión de la literatura pone de manifiesto que existen numerosos esquemas de evaluación para evaluar el contenido de mapas conceptuales. Un esquema que ha tenido bastante divulgación fue el propuesto por Novak y Gowin (1984), que evalúa tanto componentes semánticos como estructurales. En este esquema evaluativo se toman en cuenta proposiciones válidas, niveles de jerarquía, ramificaciones, enlaces cruzados y ejemplos. En este método (Cañas et al., 2006; Coffey et al., 2003), los niveles jerárquicos son reflejo del grado de subsunción entre conceptos, las ramificaciones revelan la diferenciación progresiva, mientras que los enlaces cruzados ponen de manifiesto el grado de integración del conocimiento. Sin embargo, no se tienen en cuenta otros componentes de interés como la presencia de un concepto principal o la presencia de todos los conceptos relevantes como se realiza en el método que aportan Domínguez-Marrufo et al. (2010) u otros aspectos merecedores de atención como la propuesta de análisis semántico realizada por Miller y Cañas (2008). Para salvar estas dificultades, y dadas las evidentes ventajas que supone la utilización de los mapas conceptuales para el aprendizaje y su potencial para ser utilizados en todos los niveles educativos, el presente estudio tuvo como objetivo la elaboración de un instrumento en formato rúbrica que permita a los alumnos, mediante indicadores precisos, la evaluación de la calidad tanto de sus ejecuciones como de las ejecuciones realizadas por otros.

\section{Método}

\section{Participantes}

Los participantes fueron dos grupos de 31 y 18 alumnos de la asignatura Psicología de la Educación del Grado de Educación Infantil de la Universidad de La Rioja que formaban dos Grupos Reducidos de prácticas de la asignatura. El 90,32 y el $88,88 \%$ de la muestra fueron mujeres, respectivamente.

\section{Instrumentos}

Rúbrica para la evaluación de los mapas conceptuales. A pesar de que actualmente no existe un consenso en los criterios evaluables ni en la metodología para realizar dicha evaluación, el objetivo fue salvar esta dificultad y elaborar ad hoc una rúbrica (Anexo 1) que recogiera criterios objetivables provenientes de diferentes autores (e.g., Cañas et al., 2006; Domínguez-Marrufo, 2010; Domínguez-Marrufo, Sánchez-Valenzuela y Aguilar-Tamayo, 2010; Miller y Cañas, 2008a, 2008b; Novak, 1998; Novak y Gowin, 1988).

Este instrumento de evaluación supone una guía para la lectura e interpretación de mapas conceptuales. Fue diseñado pensando tanto en el docente como en los alumnos para ayudar a ambos en el proceso de valoración de calidad de los mapas conceptuales.

En cuanto a la metodología para puntuar los mapas, de acuerdo a los criterios de la literatura especializada (e.g., Reyes, 2005) se utilizó el método de puntuación estructural que supone evaluar los mapas utilizando criterios como la identificación de 
proposiciones correctas, la presencia de niveles diferentes de jerarquía y la presencia de uniones cruzadas. El protocolo de puntuación asigna diferentes puntos a los diferentes elementos estructurales del mapa y se asigna una puntuación final.

Por un lado, para todas las categorías se estableció una puntuación ponderada y, por otro, siguiendo a Domínguez-Marrufo et al. (2010) para las cuatro primeras categorías se determinaron asimismo cuatro niveles de desempeño, expresados en una escala tipo Likert de 4 puntos (3-0), siendo el tres el nivel más alto para asignar a la ejecución de la tarea y el nivel 0, la valoración mínima (ver Anexo 1).

De acuerdo con la teoría y técnica del mapa conceptual se determinaron como categorías a evaluar: 1) el concepto principal, 2) los conceptos subordinados, 3) las proposiciones, 4) la presencia de proposiciones dinámicas, 5) conexiones cruzadas y creatividad, 6) la jerarquía y, por último 7) los ejemplos. A continuación se describen cada una de estas categorías.

Concepto principal (Domínguez-Marrufo et al., 2010). Para la valoración de los conceptos se hace distinción entre el concepto principal y los conceptos subordinados, ya que si bien todos los conceptos tienen importancia dentro del mapa, el concepto principal orienta en parte el desarrollo de la jerarquía. De acuerdo a los autores, se establecen cuatro niveles de desempeño. Por último, se ponderó esta categoría con tres puntos por cada punto asignado.

Conceptos subordinados (Domínguez-Marrufo et al., 2010). Esta categoría considera aquellos conceptos importantes según la temática. Se debe identificar si están todos o sólo algunos de éstos en el mapa conceptual. De acuerdo a los autores, se establecen cuatro niveles de desempeño. Por último, se ponderó esta categoría con 10 puntos por cada punto asignado.

Proposiciones (Modificación del criterio de Novak y Gowin, 1988). Se trata de evaluar si es válida la relación de significado entre dos conceptos mediante la línea que los une y mediante la o las palabras de enlace correspondientes. Frente al método utilizado de Novak y Gowin de sumar un punto por cada proposición válida y significativa que aparezca en el mapa, se tomó la decisión de seguir el procedimiento de Domínguez-Marrufo et al. (2010) y establecer 4 niveles de desempeño, tal y como puede observarse en el Anexo 1. Por último, se ponderó esta categoría con 10 puntos por cada punto asignado.

Presencia de proposiciones dinámicas (Miller y Cañas, 2008). Este criterio tiene como objetivo evaluar la presencia de proposiciones que impliquen movimiento, acción, cambio de estado o relaciones de dependencia. Este tipo de proposiciones suponen una elaboración más compleja de la información que proposiciones estáticas (que describen, definen). Se consideró muy interesante la inclusión de este criterio ya que implicaba una evaluación cualitativa de la calidad del mapa conceptual. Se siguió asimismo el procedimiento de Domínguez-Marrufo et al. (2010) para este criterio y se establecieron 4 niveles de desempeño. Por último, se ponderó esta categoría con 10 puntos por cada punto asignado.

Conexiones cruzadas y creatividad (Adaptación del criterio de Novak y Gowin, 1988). Se trata de evaluar si el mapa muestra conexiones significativas entre los distintos segmentos de la jerarquía conceptual y si es válida esta relación. Se asume la propuesta de los autores para otorgar 10 puntos por cada conexión cruzada válida y 
significativa. Los autores insisten en que las conexiones cruzadas creativas o singulares pueden ser objeto de un reconocimiento especial o recibir una puntuación adicional, pero no establecen dicha puntuación. El equipo investigador tomó la decisión de calificar con cinco puntos adicionales cada conexión cruzada creativa.

Jerarquía. Este criterio, asumido del trabajo original de Novak y Gowin (1988) persigue evaluar si el mapa presenta una estructura jerárquica (si cada uno de los conceptos subordinados es más específico y menos general que el concepto que hay dibujado sobre él) y la complejidad de dicha jerarquía mediante el número de niveles jerárquicos válidos. Los autores adjudican cinco puntos a cada nivel jerárquico válido.

Ejemplos (Novak y Gowin, 1988). Finalmente, se evalúa la aportación por parte del estudiante de ejemplos válidos (un punto cada ejemplo) que enriquezcan el mapa conceptual.

\section{Procedimiento}

El estudio se llevó a cabo en la asignatura Psicología de la Educación del Grado de Educación Infantil de la Universidad de La Rioja. Esta asignatura tiene carácter obligatorio y se desarrolla a través de 6 créditos ECTS. La formación en elaboración y evaluación en mapas conceptuales se impartió en sesiones lectivas dedicadas al trabajo en grupos reducidos (grupos de prácticas) y tuvo una duración de cuatro horas (dos sesiones en dos semanas consecutivas). El material utilizado para ambos grupos estuvo basado en Iraizoz y González (2003).

En el primer grupo $(n=31)$ el objetivo fue obtener dos ejecuciones de los alumnos para que pudieran ser evaluadas por expertos y conocer así la fiabilidad interjueces del instrumento de medida. El procedimiento seguido fue plantear en la primera sesión una introducción a los mapas conceptuales (definición de conceptos clave, procedimiento para la elaboración de mapas conceptuales) y se efectuó la primera ejecución individual por parte de los alumnos (realización del primer mapa, presentación de la rúbrica de evaluación y autoevaluación de la ejecución). La sesión finaliza con el trabajo sobre ejemplos de ejecución de mapas conceptuales realizados por niño. En la segunda sesión se realiza la segunda ejecución individual de un mapa conceptual (realización del mapa y autoevaluación de los alumnos a través de la rúbrica). Igualmente, se trabaja sobre ejemplos de mapas conceptuales realizados por niños. Esta segunda sesión finaliza con la presentación de experiencias reales con mapas conceptuales en centros de Educación Infantil y Primaria. Como resultado el investigador obtuvo dos ejecuciones de cada alumno que servirían para el análisis de la fiabilidad interjueces.

En el segundo grupo $(n=18)$ el objetivo fue conseguir evidencias de validez del instrumento $y$, por tanto, el procedimiento seguido fue plantear en la primera sesión una introducción a los mapas conceptuales (definición de conceptos clave, procedimiento para la elaboración de mapas conceptuales) y aunque se efectuó la primera ejecución individual por parte de los alumnos y se presentó la rúbrica de evaluación, los alumnos evaluaron un mapa conceptual realizado por un niño (heteroevaluación). El mapa cumplía unas condiciones que lo hacían ser evaluado por un experto como un mapa de alta calidad. En la segunda sesión los alumnos siguen trabajando 
mediante la ejecución de mapas conceptuales pero utilizan la rúbrica para la evaluación de un mapa conceptual realizado por un niño (heteroevaluación) cuyas características lo hacían ser evaluado por un experto como un mapa de baja calidad. Para este segundo grupo la intervención finaliza igualmente con la presentación de experiencias reales con mapas conceptuales en centros de Educación Infantil y Primaria.

\section{Análisis de datos}

En primer lugar, se calculó la fiabilidad interjueces mediante la W de Kendall. En segundo lugar, el objetivo fue encontrar una evidencia de validez de la rúbrica. Dado que los dos mapas evaluados por los alumnos diferían en su calidad (el primer mapa era una buena ejecución mientras que el segundo presentaba diversos errores), se Ilevaron a cabo análisis multivariados de la varianza (MANOVA) para confirmar si los alumnos eran capaces de discriminar entre ambas ejecuciones de manera correcta. Se tomó como factor intrasujetos el mapa (alta y baja calidad) y como variables dependientes las diferentes categorías o indicadores de la rúbrica y la puntuación total. Se utilizó el valor Lambda de Wilks para observar si existían diferencias significativas entre las variables dependientes. Como índice de tamaño del efecto se empleó el estadístico eta cuadrado parcial $\left(\eta^{2}\right.$ parcial). Para el análisis de los datos se utilizó el programa estadístico SPSS 15.0 (Statistical Package for the Social Sciences, 2006).

\section{Resultados}

Respecto a la fiabilidad del instrumento diseñado, tal y como aparece en la Tabla 1 , los valores resultantes del análisis de la fiabilidad interjueces mediante la $W$ de Kendall fueron significativos y buenos $(0,74$ y 0,68 , respectivamente), para ambas ejecuciones.

Tabla 1. Fiabilidad interjueces $(n=3)$

para dos ejecuciones y una muestra de 31 alumnos

\begin{tabular}{lcccc} 
& W de Kendall & $\chi^{2}$ & gl & Sig. asintótica \\
\hline Ejecución 1 &, 74 & 66,73 & 30 & $<, 001$ \\
Ejecución 2 &, 68 & 61,64 & 30 & $<, 01$ \\
\hline
\end{tabular}

En la Tabla 2 se presentan los estadísticos descriptivos para las categorías a evaluar de los mapas conceptuales y el resultado del MANOVA. Los valores resultantes del análisis revelaron, tal y como se esperaba, diferencias estadísticamente significativas en todas las dimensiones evaluadas salvo en la categoría de ejemplos indicando que el instrumento diseñado permitía la clasificación de mapas conceptuales en función de su calidad. Los datos indicaron que los alumnos evaluaban con puntuaciones estadísticamente superiores el mapa de alta calidad que el mapa de baja calidad.

Asimismo, los índices de tamaño del efecto mostraron valores de moderados a altos indicando la elevada significación práctica de estos resultados. 
Tabla 2. MANOVA para la comparación de puntuaciones entre mapas (alta y baja calidad) mediante la utilización de la rúbrica $(n=18)$

\begin{tabular}{lccccc}
\hline Variable dependiente & $\mathbf{M 1} \boldsymbol{M}(\boldsymbol{D} \boldsymbol{t})$ & $\mathbf{M} 2 \mathbf{M}(\mathbf{D} \boldsymbol{t})$ & $\boldsymbol{F}$ & $\boldsymbol{p}$ & $\eta^{2}$ parcial \\
\hline Concepto principal & $9,00(, 00)$ & $8,33(1,28)$ & 4,85 &, 04 &, 22 \\
Conceptos subordinados & $29,44(2,35)$ & $22,22(8,78)$ & 13,74 &, 002 &, 45 \\
Proposiciones & $28,89(3,23)$ & $19,44(6,39)$ & 30,51 &, 00 &, 64 \\
Proposiciones dinámicas & $30,00(, 00)$ & $9,44(11,61)$ & 56,35 &, 00 &, 77 \\
Conexiones cruzadas & $42,22(10,60)$ & $3,88(5,01)$ & 272,15 &, 00 &, 94 \\
Conexiones creativas & $13,33(12,00)$ & $4,44(4,81)$ & 8,11 &, 01 &, 32 \\
Jerarquía & $18,06(8,59)$ & $13,05(5,72)$ & 6,37 &, 02 &, 27 \\
Ejemplos & $1,28(1,67)$ & $1,11(1,84)$ &, 16 &, 69 &, 01 \\
Total & $170,33(13,81)$ & $81,94(20,38)$ & 333,31 & 0,00 &, 95 \\
\hline
\end{tabular}

Nota. M1 = Mapa alta calidad; M2 = Mapa baja calidad

\section{Discusión}

El objetivo del estudio consistía en el desarrollo de un instrumento para la evaluación de los mapas conceptuales en formato rúbrica. De esta manera se conseguía que los alumnos poseyeran una herramienta que permitiera, mediante indicadores precisos, la evaluación de la calidad tanto de sus ejecuciones como de las ejecuciones realizadas por otros.

Los resultados indicaron que el instrumento es fiable y que muestra evidencias de validez discriminante.

Por un lado, los resultados indican que la evaluación de la calidad de los mapas ejecutados por alumnos por parte de tres jueces expertos e independientes es fiable. El coeficiente de concordancia $W$ de Kendall (que es un índice de la divergencia entre el acuerdo efectivo mostrado en los datos y el máximo acuerdo posible) mostraba valores adecuados (Siegel y Castellan, 1988). La aplicación de la rúbrica realizada por tres jueces, usuarios de mapas y con conocimiento acerca de sus fundamentos teóricos y con experiencia docente, permitió observar que aun cuando existieron variaciones de puntajes entre jueces, éstos coincidieron en otorgar puntuaciones similares a los mapas conceptuales de los alumnos. Estos resultados son similares a los observados por estudios anteriores (Domínguez-Marrufo, 2010; Domínguez-Marrufo et al., 2010).

Por otro lado, el instrumento indica evidencias de validez puesto que su utilización permite a los alumnos la discriminación entre mapas que difieren en su calidad. Los alumnos, mediante la utilización de la rúbrica diseñada, fueron capaces de discriminar entre ejecuciones de alta y baja calidad no solo a nivel general sino en todas las categorías. El instrumento diseñado permitió a los alumnos discriminar la calidad de los mapas en todas las dimensiones evaluadas salvo en la categoría de ejemplos indicando la validez del instrumento. 
Respecto a la categoría de ejemplos, un análisis posterior de los mapas evaluados reveló la escasa frecuencia de ejemplos y por tanto la inexistencia de diferencias estadísticamente significativas pudo deberse a este hecho.

En cualquier caso, los índices de tamaño del efecto mostraron valores de moderados a altos indicando la elevada significación práctica de estos resultados.

\section{REFERENCIAS BIBLIOGRÁFICAS}

Ausubel, D. P., Novak, J. D. y Hanesian, H. (1987). Psicología educativa. Un punto de vista cognoscitivo. México: Trillas.

Cañas, A. J., Novak, J., Miller, N. L., Collado, C., Rodríguez, M., Concepción, M., Santana, C. y Peña, L. (2006). Confiabilidad de una taxonomía topológica para mapas conceptuales. En Second International Conference on Concept Mapping: September 5-8 2006. San José, Costa Rica: Universidad de Costa Rica.

Domínguez-Marrufo, L. S. (2010). Rúbrica y Puntaje del Mapa Conceptual. Una propuesta para la evaluación de mapas conceptuales en la educación Superior. (Tesis de maestría no publicada). Universidad Autónoma del Estado de Morelos, México.

Domínguez-Marrufo, L. S., Sánchez-Valenzuela, M. M. y Aguilar-Tamayo, M. F. (2010). Rúbrica con sistema de puntaje para evaluar mapas conceptuales de lectura de comprensión. En J. Sánchez, A. J. Cañas y J. D. Novak (Eds.), Concept maps: Making learning meaningful (pp. 210-213). Chile: Lom Ediciones.

González, F. M. (1997). Diagnosis of Spanish Primary School Students' Common Alternative Science Conceptions. School, Science and Mathematics, 2, 68-74.

González, F. M. e Iraizoz, N. (1995). Errores Conceptuales en Alumnos de Enseñanza Primaria: Implicaciones Educativas. Estudios de Psicología y Pedagogía, 7, 67-116.

González, F. M., Moron, C. y Novak, J. D. (2001). Errores conceptuales. Diagnosis, tratamiento y reflexiones. Pamplona: Eunate.

Iraizoz, N. y González, F. (2003). El mapa conceptual: un instrumento apropiado para comprender textos expositivos. Pamplona: Blitz.

Luque, A., Molina, A. y Ontoria, A. (2003). Los mapas conceptuales, una técnica para mejorar las capacidades cognitivas y sociopersonales. Revista de Ciencias de la Educación, 194, 207-224.

Miller, N. L. y Cañas, A. J. (2008a). A semantic scoring rubric for concept maps: design and reliability. En Third International Conference on Concept Mapping, sept. 22-25, 2008. Tallinn, Estonia: Tallinn University.

Miller, N. L. y Cañas, A. J. (2008b). Effect of the nature of the focus question on presence of dynamic propositions in a concept map. En Third International Conference on Concept Mapping, sept. 22-25, 2008. Tallinn, Estonia: Tallinn University.

Novak, J. D. (1998). Conocimiento y aprendizaje: Los mapas conceptuales como herramientas facilitadoras para escuelas y empresas. Madrid: Alianza.

Novak, J. D. y Gowin, D. B. (1988). Aprendiendo a aprender. Barcelona: Martínez Roca.

Novak, J. D. y Musonda, D. (1991). A twelve-year longitudinal study of science concept learning. American Educational Research Journal, 28, 117-153. 
Ontoria, A. (1992). Mapas conceptuales una técnica para aprender. España: Narcea. Pascual, M. T. y Pérez-Albéniz, A. (2006). Enseñar a pensar en el aula universitaria. Una experiencia en las aulas de maestro de la Universidad de La Rioja. Contextos Educativos, 13, 141-154. DOI: http://doi.org/10.18172/con.631.

Pérez-Albéniz, A., Lucas-Molina, B., Solbes-Canales, I., Calderón, S., y MartínSeoane, G. (2016). Los mapas conceptuales como estrategia de aprendizaje en la enseñanza universitaria. Innovación Educativa, 26, 233-243. DOI: http://doi. org/10.15304/ie.26.3449.

Reyes, M. (2005). Uso de los mapas conceptuales en química. Mérida: Escuela Venezolana de Enseñanza en Química.

Siegel, S. y Castellan, N. J. (1988). Nonparametric statistics for the behavioral sciences. New York: McGraw-Hill.

Statistical Package for the Social Sciences (2006). SPSS Base 15.0 User's Guide. Chicago, IL: SPSS Inc. 


\section{Anexo 1}

\begin{tabular}{|c|c|c|c|c|c|c|c|}
\hline $\begin{array}{l}\text { CRITERIOS } \\
\text { EVALUABLES }\end{array}$ & 3 & 2 & 1 & 0 & Puntuación & Peso & Total \\
\hline $\begin{array}{l}\text { 1. Concepto } \\
\text { principal }\end{array}$ & $\begin{array}{l}\text { El concepto } \\
\text { principal es } \\
\text { adecuado y } \\
\text { pertinente con } \\
\text { el tema que se } \\
\quad \text { trata. }\end{array}$ & $\begin{array}{l}\text { El concepto } \\
\text { principal es } \\
\text { relevante dentro } \\
\text { del tema pero } \\
\text { puede ser auxiliar } \\
\text { y/o no representa } \\
\text { el objetivo } \\
\text { principal. }\end{array}$ & $\begin{array}{l}\text { El concepto } \\
\text { principal } \\
\text { pertenece al } \\
\text { tema pero no es } \\
\text { fundamental }\end{array}$ & $\begin{array}{l}\text { El concepto } \\
\text { principal no } \\
\text { tiene relación } \\
\text { con el tema ni } \\
\text { representa al } \\
\text { tema tratado. }\end{array}$ & & $\times 3$ & \\
\hline $\begin{array}{l}\text { 2. Conceptos } \\
\text { subordinados }\end{array}$ & $\begin{array}{l}\text { Incluye todos } \\
\text { los conceptos } \\
\text { importantes que } \\
\text { representan la } \\
\text { información } \\
\text { principal del } \\
\text { tema. } \\
\text { No repite } \\
\text { conceptos. }\end{array}$ & $\begin{array}{l}\text { Incluye la mayoría } \\
\text { de los conceptos } \\
\text { importantes que } \\
\text { representan la } \\
\text { información } \\
\text { principal sobre el } \\
\text { tema. } \\
\text { No repite } \\
\text { conceptos. }\end{array}$ & $\begin{array}{c}\text { Faltan la mayoría } \\
\text { de los conceptos } \\
\text { importantes que } \\
\text { representan la } \\
\text { información } \\
\text { principal del } \\
\text { tema. } \\
\text { Repite algún } \\
\text { concepto. }\end{array}$ & $\begin{array}{l}\text { Incluye solo } \\
\text { algunos de } \\
\text { los conceptos } \\
\text { importantes } \\
\text { que representan } \\
\text { lo importante } \\
\text { del tema, pero } \\
\text { faltan los más } \\
\text { significativos. } \\
\text { Repite varios } \\
\text { conceptos } \\
\text { y/o aparecen } \\
\text { varios ajenos o } \\
\text { irrelevantes. }\end{array}$ & & X 10 & \\
\hline $\begin{array}{l}\text { 3. Proposiciones } \\
\text { ¿Se indica la } \\
\text { relación de } \\
\text { significado entre } \\
\text { dos conceptos } \\
\text { mediante la } \\
\text { línea que los } \\
\text { une y mediante } \\
\text { la(s) palabra(s) } \\
\text { de enlace } \\
\text { correspondiente(s)? } \\
\text { ¿Es válida esta } \\
\text { relación? }\end{array}$ & $\begin{array}{l}\text { La mayor } \\
\text { parte de las } \\
\text { proposiciones } \\
\text { son válidas de } \\
\text { acuerdo al tema } \\
\text { y representan } \\
\text { la información } \\
\text { principal. }\end{array}$ & $\begin{array}{l}\text { Algunas de las } \\
\text { proposiciones } \\
\text { son inválidas o } \\
\text { no representan } \\
\text { la información } \\
\text { principal de } \\
\text { acuerdo al tema. }\end{array}$ & $\begin{array}{l}\text { Sólo algunas de } \\
\text { las proposiciones } \\
\text { son válidas de } \\
\text { acuerdo al tema o } \\
\text { Presenta varias } \\
\text { proposiciones } \\
\text { irrelevantes o muy } \\
\text { generales. }\end{array}$ & $\begin{array}{c}\text { Presenta } \\
\text { proposiciones } \\
\text { inválidas de } \\
\text { acuerdo al tema } \\
\text { con enlaces } \\
\text { que describen } \\
\text { una relación } \\
\text { inexistente o } \\
\text { afirmaciones } \\
\text { falsas. } \\
\text { Presenta } \\
\text { afirmaciones } \\
\text { vagas y/o más } \\
\text { de una línea } \\
\text { sin palabras de } \\
\text { enlace. }\end{array}$ & & X 10 & \\
\hline $\begin{array}{l}\text { 4. Presencia de } \\
\text { proposiciones } \\
\text { dinámicas. } \\
\text { Implican:1) } \\
\text { movimiento, 2) } \\
\text { acción, 3) cambio } \\
\text { de estado, o 4) } \\
\text { relaciones de } \\
\text { dependencia. Se } \\
\text { dividen en causales } \\
\text { (uno de los } \\
\text { conceptos es causa } \\
\text { y el otro efecto de } \\
\text { manera clara) y no } \\
\text { causales. } \\
\text { Las causales } \\
\text { puede ser además } \\
\text { cuantificables } \\
\text { (especifican la } \\
\text { manera en la que } \\
\text { cierto cambio } \\
\text { en un concepto } \\
\text { provoca un cambio } \\
\text { en otro). }\end{array}$ & $\begin{array}{c}\text { Contiene } \\
\text { más de dos } \\
\text { proposiciones } \\
\text { dinámicas } \\
\text { causales (por } \\
\text { ejemplo, el } \\
\text { tabaco puede } \\
\text { provocar } \\
\text { cáncer) o alguna } \\
\text { proposición } \\
\text { dinámica causal } \\
\text { cuantificable } \\
\text { (por ejemplo, } \\
\text { la mejora de } \\
\text { la calidad en } \\
\text { educación } \\
\text { contribuye a un } \\
\text { mayor desarrollo } \\
\text { nacional, el } \\
\text { incremento en } \\
\text { la transparencia } \\
\text { de las cuestiones } \\
\text { públicas } \\
\text { desalienta la } \\
\text { corrupción). }\end{array}$ & $\begin{array}{c}\text { Contiene } 1 \text { o } 2 \\
\text { proposiciones } \\
\text { dinámicas } \\
\text { causales. } \\
\text { Ejemplos: la carga } \\
\text { eléctrica genera } \\
\text { campos eléctricos, } \\
\text { la reproducción } \\
\text { permite la } \\
\text { continuidad de las } \\
\text { especies. }\end{array}$ & $\begin{array}{l}\text { Solo contiene } \\
\text { proposiciones } \\
\text { dinámicas } \\
\text { no causales. } \\
\text { Ejemplos: las } \\
\text { raíces absorben } \\
\text { el agua, los } \\
\text { herbívoros comen } \\
\text { plantas, los seres } \\
\text { vivos necesitan } \\
\text { oxígeno. }\end{array}$ & $\begin{array}{l}\text { No contiene } \\
\text { ningún tipo de } \\
\text { proposiciones } \\
\text { dinámicas. } \\
\text { Solo incluye } \\
\text { proposiciones } \\
\text { estáticas (que } \\
\text { describen, } \\
\text { definen), como } \\
\text { por ejemplo: } \\
\text { el sol es una } \\
\text { estrella, España } \\
\text { está ubicada } \\
\text { en Europa, los } \\
\text { animales pueden } \\
\text { ser vertebrados. }\end{array}$ & & $\mathrm{X} 10$ & \\
\hline
\end{tabular}




\begin{tabular}{|c|c|c|}
\hline $\begin{array}{l}\text { 5. Conexiones } \\
\text { cruzadas y } \\
\text { creatividad. } \\
\text { ¿Muestra el mapa } \\
\text { conexiones } \\
\text { significativas }\end{array}$ & Número de conexiones cruzadas & $\times 10$ \\
\hline $\begin{array}{l}\text { segmentos de } \\
\text { la jerarquía } \\
\text { conceptual? ¿Es } \\
\text { significativa y } \\
\text { válida la relación } \\
\text { que se muestra? }\end{array}$ & Número de conexiones creativas & X 5 \\
\hline $\begin{array}{l}\text { 6. Jerarquía } \\
\text { ¿Presenta el mapa } \\
\text { una estructura } \\
\text { jerárquica? ¿Es } \\
\text { cada uno de } \\
\text { los conceptos } \\
\text { subordinados más } \\
\text { específico y menos } \\
\text { general que el } \\
\text { concepto que hay } \\
\text { dibujado sobre él? }\end{array}$ & Número de niveles jerárquicos válidos & $\times 5$ \\
\hline 7. Ejemplos & Un punto por cada ejemplo válido (no debe estar rodeado ya que no es un concepto) & $\mathrm{X} 1$ \\
\hline
\end{tabular}

